\begin{tabular}{|c|l|}
\hline Title & Characterization of mouse mediastinal fat- associated lymphoid clusters \\
\hline Author(s) & Elewa, Y aser Hosny A li; Ichii, Osamu; Otsuka, Saori; Hashimoto, Y oshiharu; Kon, Y asuhiro \\
\hline Citation & $\begin{array}{c}\text { Cell and Tissue Research, 357(3), 731-741 } \\
\text { https://doi.org/10.1007/300441-014-1889-6 }\end{array}$ \\
\hline Issue Date & 201409 \\
\hline Doc URL & http://hdl.handle.net/2115/59758 \\
\hline Rights & The final publication is available at link.springer.com \\
\hline Type & article (author version) \\
\hline Additional Information & There are other files related to this item in HUSCAP. Check the above URL. \\
\hline CellTissueRes v.357p.731.pdf
\end{tabular}

Instructions for use 


\section{Characterization of mouse mediastinal fat-associated lymphoid clusters}

2 Yaser Hosny Ali Elewa ${ }^{1,2}$, Osamu Ichii $^{2}$, Saori Otsuka ${ }^{2}$, Yoshiharu Hashimoto ${ }^{3}$, Yasuhiro Kon ${ }^{2, *}$

3

4

5

6
${ }^{1}$ Department of Histology and Cytology, Faculty of Veterinary Medicine, Zagazig University, Zagazig 44519, Egypt

${ }^{2}$ Laboratory of Anatomy, Department of Biomedical Sciences, Graduate School of Veterinary Medicine, Hokkaido University, Sapporo 060-0818, Japan

${ }^{3}$ Office for International Accreditation of Veterinary Education, Obihiro University of Agriculture and Veterinary Medicine, Obihiro 080-8555, Japan

\footnotetext{
*Corresponding author: Yasuhiro Kon, DVM, PhD, Laboratory of Anatomy, Department of
} Biomedical Sciences, Graduate School of Veterinary Medicine, Hokkaido University, Kita 18, Nishi
9, Kita-ku, Sapporo 060-0818, Japan. y-kon@vetmed.hokudai.ac.jp 


\section{Abstract}

The association between adipose tissue and immunity has been established, and fat-associated

lymphoid clusters (FALCs) are considered a source of immune cells. We discovered lymphoid

clusters (LCs) in mouse mediastinal fat tissues (MFTs). In three mice strains, including Th1-biased

C57BL/6N (B6), Th2-biased DBA/2Cr (DBA), and autoimmune-prone MRL/MpJ (MRL), LCs

without a fibrous capsule and germinal center were observed in white-colored MFTs extending from the diaphragm to the heart. The number and size of the LCs were larger in 12-month-old mice than in 3-month-old mice in all of the examined strains. Moreover, B6 had an especially large number of LCs compared with DBA and MRL. The immune cells in the LCs consisted of mainly T-cells and some B-cells. The majority of T-cells were CD4+ helper T (Th) cells, rather than CD8+ cytotoxic T-cells, and there was no obvious immune cell population difference among three mouse strains. Furthermore, high endothelial venules and lymphatic vessels were well developed in the LCs of B6 mice than other strains. Interestingly, few CD133+ hematopoietic progenitor cells and few c-Kit+/CD127+ natural helper cells were detected in the LCs. BrdU+ proliferating cells were abundantly detected in the LCs of B6 mice compared to other strains and tended to increase with ages. This is the first report of LCs in mouse MFTs and we suggest that the size and number of LCs are affected by the mouse genetic background. We termed the LCs mediastinal fat-associated lymphoid clusters (MFALCs). These clusters may be considered niches for Th cell production. 
Key words: mediastinal, adipose tissue, lymphoid cluster 

cushion and stores lipids as energy sources. Furthermore, adipose tissue has been recognized as a major endocrine organ, producing hormones and adipokines such as leptin, estrogen, resistin, and tumor necrosis factor alpha (Kershaw and Flier 2004). Recently, lymphoid clusters (LCs) were observed in the adipose tissue of human and mouse mesentery; they were termed fat-associated lymphoid clusters (FALCs) (Moro et al. 2010). FALCs are in direct contact with adipocytes, lack fibrous capsules, and contain T-cells, B-cells, macrophages, and dendritic cells and are also found around the kidney and genital organs (Koyasu and Moro 2011). They are structurally similar to the milky spot in the omentum, which is considered a gateway of cells between the circulation and the peritoneal cavity (Cranshaw and Leak 1990). However, unlike the milky spot, T-cell and B-cell zones or germinal center structures are not observed in FALCs (Moro et al. 2010). Results from retinoic acid receptor-related orphan receptor gamma $(\mathrm{ROR} \gamma)$-deficient mice and alymphoplasia (aly/aly) mice suggested that the differentiation pathway of FALCs is distinct from that of lymph nodes or Peyer's patches (Nishikawa et al. 2003).

51 There are 2 major subsets of helper T (Th) cells, Th1 and Th2 cells, differentiated by the pattern of cytokine production (Fiorentino et al. 1989). Th1 cytokines include interleukin 2 (IL-2), IL-12, and interferon gamma (IFN- $\gamma$ ), which are associated with the activation of cellular immunity. Th2 
cytokines include IL-10, IL-4, IL-5, and IL-6, which are associated with humoral immunity. In addition to these Th cells, Th17 cells producing IL-17 have been identified, and these cells are considered critical for the development of autoimmune disease (Bettelli et al. 2007). against infection (Zygmunt and Veldhoen 2011). B-cells and T-cells are the major types of cells involved in the adaptive or acquired immune system. In addition to two prototypic innate lymphocyte populations, namely classical natural killer (NK) and lymphoid tissue inducer (LTi) cells, recent studies have identified Th2-type innate lymphocytes, such as Nh cells (Moro et al. 2010 and Koyasu and Moro 2011). Interestingly, such novel Th2-type innate lymphocyte, natural helper $(\mathrm{Nh})$ cells express c-Kit, Sca-1, interleukin-2 receptor (IL-2R), interleukin-7 receptor (IL-7R), and IL-33R were found in the FALC. Nh cells in the FALC produce Th2 cytokines, including IL-5 and IL-13, and play important roles in innate and anti-helminthes immunity (Moro et al. 2010). The IL-7R consists of two chains, the IL-7R $\alpha$-chain (IL-7R $\alpha$; also known as CD127) and the common cytokine-receptor $\gamma$-chain $(\gamma \mathrm{c}$; also known as CD132) (Mazzucchelli and Durum, 2007). Recent studies revealed that CD127 is also expressed by mouse natural killer (NK) lineage cells early during differentiation (Chiossone et al. 
autoantibody production, lymphadenopathy, and glomerulonephritis associated with Th1 responses,

similar to human systemic lupus erythematosus. In contrast, abnormal Th2 skewing in MRL/lpr

altered the feature of nephritis from proliferative to membranous glomerulonephritis, with an altered

balance of Th1 (IFN- $\gamma$ ) and Th2 (IL-4) cytokine production (Shimizu et al. 2005). Shimizu et al.

reported that the imbalance of Th responses is associated with autoimmune phenotypes. Interestingly,

it has been reported that the genetic background of mice influences the Th responses. Briefly, mouse

strains favoring a Th1 response include C57BL/6, C57BL/10, and B10.D2/nSn, and those favoring a

Th2 response include DBA/2, BALB/c, BALB/cBy, BALB.B, and BALB.K (Linda et al. 2000;

Charles et al. 2000). These differences in Th responses are caused by differences in the genetic background of the mice, and the susceptibility to several diseases, such as skin tumor, pneumonia, and obesity, differs between C57BL/6 and DBA/2 (Gudmundsson et al. 1998; Butler et al. 2002). them mediastinal fat-associated lymphoid clusters (MFALCs). Furthermore, the size and lymphocyte number of the MFALCs differed among the studied mouse strains: Th1-biased C57BL/6, Th2-biased

$\mathrm{DBA} / 2$, and autoimmune-prone MRL/MpJ. This is the first report of strain-specific differences in characteristics of the immune responses in these strains. 

C57BL/6N (B6), DBA/2Cr (DBA), and MRL/MpJ (MRL) mice were used in the present study. Eight- to ten-week-old mice purchased from Japan SLC (Hamamatsu, Shizuoka, Japan) were maintained with free access to specific pathogen-free food and water in our facility and were used at 3 and 12 months of age. In handling the experimental animals, the investigators adhered to the Guide for the Care and Use of Laboratory Animals, Hokkaido University, Graduate School of Veterinary Medicine (approved by the Association for Assessment and Accreditation of Laboratory Animal Care International).

After euthanasia by deep inhalation anesthesia, MFTs within the mediastinum extending from the caval foramen of the diaphragm to the heart and the thymus were removed and fixed with $4 \%$ paraformaldehyde (Fig. 1). After overnight fixation, specimens were washed in distilled water, penetration by glycerol. For histological examination, the removed MFTs were dehydrated in graded alcohol and embedded in paraffin. Subsequently, 3- $\mu \mathrm{m}$ paraffin sections of MFT were deparaffinized, rehydrated, stained with hematoxylin and eosin (HE), and observed by light microscopy. 
111 Using the MFT sections, immunohistochemical analysis for B220, BrdU, CD3, CD4, CD8,

112 CD133, peripheral node addressin (PNAd), and lymphatic vessel endothelial hyaluronic acid

113 receptor 1 (LYVE-1) was performed to detect B-cells, pan T-cells, Th cells, cytotoxic T (Tc) cells,

114 hematopoietic progenitor cells (HPC), high endothelial venules (HEV), and lymphatic endothelial

115 cells, respectively. The immunohistochemical procedures were performed according to previous

116 reports (Elewa et al. 2010a).

117 The details of the antigen retrieval methods as well as the sources and dilutions of the

118 antibodies are listed in Table 1. For the detection of proliferating cells, 5-bromo-2-deoxyuridine

119 (BrdU) (Wako Pure Chemical Industries, Tokyo, Japan) was dissolved in PBS and injected

120 intraperitoneally into mice at $100 \mathrm{mg}$ per $\mathrm{kg}$ of body weight. The MFTs were removed from the

121 animals $2 \mathrm{hr}$ after BrdU injection. To detect BrdU-labeled cells, the MFT paraffin sections were

122 stained with rat anti-BrdU antibody (Table 1). The immunopositive reactions were developed in

$1233,3^{\prime}$-diaminobenzidine- $\mathrm{H}_{2} \mathrm{O}_{2}$ solution. The sections were counterstained with hematoxylin. Sections

124 of spleen were used as positive controls and stained by different antibodies simultaneously with the

125 MFTs. The sections for negative controls were stained with isotype control Ig at the same not shown). 
Immunofluorescence

Immunofluorescence for c-Kit and CD127 was also performed to detect the $\mathrm{Nh}$ cells. The deparaffinized sections were treated with $10 \mathrm{mM}$ citrate buffer ( $\mathrm{pH} 6.0$ ) for $20 \mathrm{~min}$ at $105^{\circ} \mathrm{C}$, treated with normal donkey serum, and incubated at $4^{\circ} \mathrm{C}$ overnight with goat anti-c-Kit antibody (1:100) and rabbit anti-CD127 antibody (1:1000) (Table 1). The sections were then incubated with Alexa Fluor 488-labeled donkey anti-goat IgG and Alexa Fluor 546-labeled donkey anti-rabbit IgG (1:500; Life Technologies) for 30 min, followed by Hoechst33342 (1:2000; Dojindo, Kumamoto, Japan) for $3 \mathrm{~min}$. The immunofluorescence signals were examined by confocal microscopy.

\section{Histoplanimetry}

Light micrographs of 5 whole-mount specimens as well as paraffin sections of the MFTs from each strain were prepared for histoplanimetry. In the digital images of the sections, the areas of the LCs were observed as dark regions in the MFTs. LCs and MFTs were measured using the ImageJ software (ver. 1.32j, http://rsb.info.nih.gov/ii), and the LC-to-MFT ratio was calculated (as reported in a previous study by Elewa et al., 2010b). Based on the immunohistochemical study, the numbers of B220-, CD3-, CD4-, and CD8-positive cells in the LCs were counted in 3 different sections of the MFTs from each mouse strain. In each section, the number of immunonegative cells was also counted, and the percent of immunopositive cells among total cells was calculated as the positive 
146 cell index for each cell population. The average of the indices for each cell population was

147 presented.

148

149

Statistical analysis

150

All numerical results were shown as the mean \pm standard error (SE). The results of different

151

groups were compared using analysis of variance (ANOVA). We used the Tukey's post-hoc test for

multiple comparisons when a significant difference was observed by ANOVA $(p<0.05)$.

153 


\section{Results}

The MFTs were observed as white-colored fat tissue in the mediastinum, extending from the

caval foramen of the diaphragm to the heart (Fig. 1). The localization of the MFTs was not significantly different among the examined mice (data not shown). In the stereomicroscopic observations of the whole-mount hematoxylin specimens, the examined MFTs from all mice showed dark-stained regions that varied in shape and size (Fig. 2a-f), and these regions were confirmed as LCs by subsequent histological examination (Fig. 3). In particular, the MFTs of B6 mice (Fig. 2a) had a larger number of LCs compared with DBA and MRL mice (Fig. 2b and c). The size of these LCs was also greater in C57BL/6N mice (Fig. 2d) than in other mice strains (Fig. 2e and f). These observations were confirmed by histoplanimetry (Fig. 2g); the ratios of LC area to total MFT area were significantly higher in B6 mice than in other mice strains at 3 and 12 months; notably, the values at 12 months trended higher than the values at 3 months for all examined mice.

In the HE-stained MFT sections, the dark regions of the whole-mount MFT specimens were observed as clusters of mononuclear cells (Fig 3a-f). We termed these structures MFALCs. All examined MFALCs differed from the structure of the lymph node, neither surrounded by fibrous capsules nor formed by lymphatic nodules (Fig. 3d-f). Although MFALCs were observed in all examined mice, the size and number of MFALCs were more prominent in B6 mice than in DBA and 
MRL mice. Similar to the light microscope observations of the HE-stained MFT sections, the histoplanimetrical measurements of the ratio of the LC area to the total MFT area (Fig. 3g) revealed a significantly greater ratio in B6 mice than in other mice at 3 and 12 months. In addition, the values at 12 months trended higher than the values at 3 months for all examined mice.

Blood vessels and lymphatic vessels in mouse MFALCs

As a characteristic of MFALCs, blood capillaries and venules were observed within the LCs (Fig. 3e and Fig. 4), and several lymphocytes and red blood cells were observed in their lumens. Some endothelial cells in the venules of the MFALCs had abundant cytoplasms and large nuclei, characteristics of HEVs (Fig. 4a and b). Based on the immunohistochemical analysis of PNAd (HEV marker), the endothelial cells of the venules of the MFALCs showed positive reactions, and the positive reactions were stronger in B6 mice (Fig. 4c) than in DBA and MRL mice (Fig. 4d and e) at 12 months. No positive reaction in HEV was observed in the serial sections stained with normal IgM controls (Fig. 4f- h).

Furthermore, lymphatic vessels positive for LYVE-1 were observed within the MFALCs (Fig. 5a-b) as well as the mediastinal fat tissue (Fig. 5c-f), whereas blood vessels containing erythrocytes were not positive for LYVE-1 (Fig. 5c). Interestingly, these lymphatic vessels were abundantly observed in B6 mice (Fig. 5a-c) compared to DBA (Fig. 5d) and MRL mice (Fig. 5e and f) at 12 
months. B220, CD3, CD4, and CD8 was performed to detect B-cells, pan T-cells, Th cells, and Tc cells, respectively. In three strains, B220-positive B-cells and CD3-positive T-cells were observed; the latter was more predominantly observed than the former (Fig. 6a, b, e, and f). For T-cells, CD4-positive Th cells (Fig. 6c and g) were more abundant than CD8-positive Tc cells (Fig. 6d and h).

These results were confirmed by histoplanimetry. The immunopositive cell index of CD3 was higher than for other markers in all examined mice, and the index of CD4 was higher than that of CD8 (Fig. 6i). No significant strain-specific difference was observed in the populations of MFALC immune cells. Furthermore, few F4/80-positive cells were detected in the MFALC, and no significant strain difference was observed (data not shown). these cells were localized within large-sized LCs (Fig. 7a), the lumen of blood vessels (Fig. 7b and c), and small-sized LCs (Fig. 7b and d). The mononuclear cells in the small-sized LCs (Fig. 7b)

217 appeared to be newly aggregated, containing more CD133-positive cells than mononuclear cells in the large-sized LCs (Fig. 7a). No positive cells were observed in the serial sections stained with 
normal IgG controls (Fig. 7e and f). cells within the MFALC of C57BL/6 at ** months (Fig. 8a-d). These results were also confirmed by immuhohistochemistry for c-Kit and CD127 (Supplementary figure 1a and b). observed within the MFALC of B6 mice (Fig. 9c and f) than DBA mice (Fig. 9a and d) at 12 months. 
Discussion

229

230

231

232

\section{LCs in the MFTs}

Adipose tissue is no longer considered an inert tissue that solely functions in energy storage.

Other important roles of adipose tissue are emerging, for example, the regulation of various physiological and pathological processes. Recently, there has been much effort to define the roles of adipokine-soluble mediators derived mainly from adipocytes in the interactions between adipose tissues and immunity (MacLaren et al. 2008). Adiponectin and leptin have emerged as the most abundant adipokines produced by adipocytes, thereby redefining adipose tissue as a key component of the endocrine and immune systems (Tilg and Moschen 2006).

Novel LCs called FALCs were discovered in the mesenteric adipose tissue of humans and mice (Moro et al. 2010). In the present study, we focused our examination of mouse MFTs at 3 and 12 months, corresponding to early and late adulthood, respectively. We discovered LCs in the MFTs of the mouse mediastinum extending from the caval foramen of the diaphragm to the heart. Our histological examinations revealed that these LCs had no fibrous capsules and contained more T-cells than B-cells. Therefore, the structural characteristics and immune cell populations of the MFALCs are similar to the mesenteric FALCs (Moro et al. 2010). Furthermore, the border between the T-cell and B-cell areas was unclear, and no germinal center was observed in the LCs of the mouse MFTs, unlike the milky spot in the omentum (Cranshaw and Leak 1990). The LCs of the 
246 MFTs were observed in healthy B6 and DBA mice as well as autoimmune-prone MRL mice. These

247 morphological characteristics and the appearance of MFALCs in healthy mice differed from the

248 tertiary lymphoid follicles and LCs present in the target tissues of various immune diseases, such as

249 synovial tissue in rheumatoid arthritis (Takemura et al. 2001). From these findings, the lymphoid

250 structures that we discovered in the mouse MFTs were clearly distinct from lymphatic nodules and

251 pathological cell infiltrations and were universally observed in different mouse strains. We termed

252 these clusters MFALCs.

253

Age- and strain-specific differences in MFALCs caused by different genetic backgrounds

The putative functions of mouse mesentery FALCs are the self-replication of peritoneal B-cells,

the induction of IgA production, and the promotion of an antiparasitic response via Th2 cytokines of

Nh cells (Moro et al. 2010; Koyasu et al. 2010; Koyasu and Moro 2011). However, the proper functions of MFALCs in mice and their presence in humans are still unknown. Importantly, in this study, strain- or age-specific changes in MFALCs were observed in mice, and the size of MFALCs increased with age. Age-related changes in immune cells are important for the response to antigen. 
leukocytes increased with age in the small intestine; much of this increase was attributed to mucosal

CD3+ T-cells. In particular, CD4+ T-cells increased significantly in the jejunum, and CD8+ T-cells increased significantly with age throughout the small intestine (Fries et al. 2011). Furthermore, age-dependent development of the duct-associated lymphoid tissue (DALT) of minor salivary glands was reported in Macaca fascicularis (crab-eating macaque); DALT may play a substantial role in the local immunosurveillance of the oral cavity (Nair et al. 1987). These findings suggest the age-related increased host responses to pathogens and native antigens.

Furthermore, the mouse strain-specific differences were clarified by examination of MFALC morphology. Briefly, the ratio of MFALC area to MFT area was significantly higher in B6 mice than in DBA mice and autoimmune-prone MRL mice. Interestingly, Th1-biased B6 mice are susceptible to hypersensitive pneumonitis, but Th2-biased DBA mice are resistant to it (Butler et al. 2002; Gudmundsson et al. 1998). Hypersensitive pneumonitis is a granulomatous inflammatory lung disease caused by repeated inhalation of organic antigens in humans and mice (Fink 1992; Sharma and Fujimura 1995). Although exposure to pathogenic antigens is widespread in humans, only a minority (5-10\%) of the exposed individuals develop clinical disease (Fink 1992), and variations in the susceptibility to hypersensitive pneumonitis, due, for example, to genetic background, might contribute to these differences in humans as well as other animals. Furthermore, eosinophilic 
macrophage pneumonia is observed more frequently in mice with the B6 genetic background

(Murray and Luz 1990; Hoenerhoff et al. 2006). In addition to lung diseases, the genetic background

of B6 mice may be associated with aseptic inflammation of the upper urinary tract, which accelerates

(Ichii et al. 2008; Ichii et al. 2011). Therefore, strain-specific differences in MFALC areas may

underlie the differential immune response, mediated by several genetic factors. In particular, the

Th1-biased B6 background might be associated with the acceleration of susceptibility to intrapleural

disease.

In the histological examination, we clarified that the majority of the immune cells in the

MFALCs were T-cells, rather than B-cells, and specifically CD4-positive Th cells, rather than

CD8-positive Tc cells. There were no strain-specific differences in these populations. The mesenteric

FALCs also contain CD3-positive T-cells and B220-positive B-cells (Moro et al. 2010). The

IL-2R ${ }^{+}, \mathrm{IL}_{-} 7 \mathrm{R}^{+}$, and IL-33R ${ }^{+}$) (Moro et al. 2010). Similarly, our results revealed the presence of $\mathrm{c}-\mathrm{Kit}^{+} / \mathrm{CD} 127^{+}$cells, and we suggested that the MFALCs contained Nh cells. Furthermore, we 
300 CD127 is also expressed by mouse natural killer cells (NK) lineage cells early during differentiation.

301 Thus, our finding of numerous $\mathrm{CD} 127^{+}$cells within the MFALCs suggest that the MFALCs could be 302 also a source of NK cells. Interestingly, we found blood vessels, such as blood capillaries and venules, in the MFALCs of 304 all examined mice, and the venules showed positive reactions for an HEV marker, PNAd. These PNAd-positive venules were more prominent in B6, which had the largest MFALC area among the examined mouse strains. Lymphocytes are intrinsically mobile; they circulate continuously between the blood and secondary lymphoid tissues. When naive lymphocytes first enter the lymph nodes and Peyer's patches, they adhere to and migrate across the HEV (Kraal and Mebius 1997; Miyasaka and Tanaka 2004). Moreover, LYVE-1-positive lymphatic vessels were observed within the MFALCs and the MFTs. Such lymphatic vessels were more prominent in B6 mice than other examined strains suggesting the major role of lymph vessels for lymphocyte emigration and antigen transport. of the blood vessels in the MFALCs, indicating the presence of HPCs (Handgretinger and Kuçi 2013). It has been suggested that the migration of hematopoietic stem cells occurs through the blood, across the endothelial vasculature, to different organs and to their bone marrow niches via a homing process (Lapidot et al. 2005). From these findings, we propose that immature hematopoietic cells migrate to MFALCs via HEV and act as niches for Th cell production. 

numerous BrdU-positive cells were observed in the B6 mice compared to other strains. Moreover,

proliferation of T-cells. The present study revealed that normal MFALC structures contain T-cells, especially on the T regulatory response.

\section{Acknowledgements}

This work was supported by the Partnership and Ownership Initiative (ParOwn), which was funded by 


\section{References}

337 Bettelli E, Oukka M, Kuchroo VK (2007) TH-17 cells in the circle of immunity and autoimmunity.

$338 \quad$ Nat Immunol 8: 345-350

Butler NS, Monick MM, Yarovinsky TO, Powers LS, Hunninghake GW (2002) Altered IL-4 mRNA

340 stability correlates with Th1 and Th2 bias and susceptibility to hypersensitivity pneumonitis in two

341 inbred strains of mice. J Immunol 169: 3700-3709

342 Charles DM, Kristi K, Jennifer MA, Michelle J (2000) M-1/M-2 macrophages and the Th1/Th2

343 paradigm. J Immunol 164: 6166-6173

344 Chiossone L, Chaix J, Fuseri N, Roth C, Vivier E, Walzer T (2009) Maturation of mouse NK cells is

a 4-stage developmental program. Blood 113: 5488-5496.

Cranshaw ML, Leak LV (1990) Milky spots of the omentum: a source of peritoneal cells in the normal and stimulated animal. Arch Histol Cytol 53: 165-177

Elewa YH, Bareedy MH, Abuel-Atta AA, Ichii O, Otsuka S, Kanazawa T, Lee SH, Hashimoto Y,

Kon Y (2010a) Cytoarchitectural differences of myoepithelial cells among goat major salivary

Elewa YH, Bareedy MH, Abuel-Atta AA, Ichii O, Otsuka S, Kanazawa T, Lee SH, Hashimoto Y, 
354 Fink JN (1992) Hypersensitivity pneumonitis. Clin Chest Med 13: 303-309

355 Fiorentino DF, Bond MW, Mosmann TR (1989) Two types of mouse T-helper cell. IV. Th2 clones

356 secrete a factor that inhibits cytokine production by Th1 clones. J Exp Med 170: 2081-2095

357 Fries PN, Popowych YI, Guan LL, Griebel PJ (2011) Age-related changes in the distribution and

358 frequency of myeloid and $\mathrm{T}$ cell populations in the small intestine of calves. Cell Immunol 271:

$359 \quad 428-437$

360 Fulton RB, Meyerholz DK, Varga SM (2010) Foxp3 ${ }^{+}$CD4 regulatory T cells limit pulmonary

361 immunopathology by modulating the $\mathrm{CD} 8 \mathrm{~T}$ cell response during respiratory syncytial virus

362 infection. J Immunol 185: 2382-2392

363 Gudmundsson G, Monick MM, Gary W (1998) IL-12 modulates expression of hypersensitivity

364 pneumonitis. J Immunol 161: 991-999

365 Handgretinger R, Kuçi S (2013) CD133-positive hematopoietic stem cells: from biology to medicine.

366 Adv Exp Med Biol 777: 99-111

367 Hoenerhoff MJ, Starost MF, Ward JM (2006) Eosinophilic crystalline pneumonia as a major cause of

368 death in 129S4/SvJae mice. Vet Pathol 43: 682-688

369 Ichii O, Otsuka S, Namiki Y, Hashimoto Y, Kon Y (2011) Molecular pathology of murine ureteritis

370 causing obstructive uropathy with hydronephrosis. PLoS ONE 6: e27783

371 Ichii O, Konno A, Sasaki N, Endoh D, Hashimoto Y, Kon Y (2008) Autoimmune glomerulonephritis 
372 induced in congenic mouse strain carrying telomeric region of chromosome 1 derived from

373 MRL/MpJ. Histol Histopathol 23: 411-422

374 Kershaw EE, Flier JS (2004) Adipose tissue as an endocrine organ. J Clin Endocrinol Metab 89:

$375 \quad 2548-2556$

376 Koyasu S, Moro K (2011) Type 2 innate immune responses and the natural helper cell. Immunology

$377 \quad 132: 475-481$

378 Koyasu S, Tanabe M, Takeuchi T, Moro K (2010) Natural helper cells: a new player in the innate

379 immune response against helminth infection. Adv Immunol 108: 21-44

380 Koyasu S, Moro K (2011) Innate Th2-type immune responses and the natural helper cell, a newly

381 identified lymphocyte population. Curr Opin Allergy Clin Immunol 11: 109-114

382 Kraal G, Mebius RE (1997) High endothelial venules: lymphocyte traffic control and controlled

383 traffic. Adv Immunol 65: 347-395

384 Lapidot T, Dar A, Kollet O (2005) How do stem cells find their way home? Blood J 106: 1901-1910

385 Linda DH, Sharon MC, Byungsuk K, Ronald B (2000) Increased severity of pseudomonas

386 aeruginosa corneal infection in strains of mice designated as Th1 versus Th2 responsive. Invest

387 Ophthalmol Vis Sci 41: 805-810

388 MacLaren R, Cui W, Cianflone K (2008) Adipokines and the immune system: an adipocentric view. 
390 Mannering SI, Zhong J, Cheers C (2002) T-cell activation, proliferation and apoptosis in primary

391 Listeria monocytogenes infection. Immunology 106: 87-95

392 Mazzucchelli R, Durum SK (2007) Interleukin-7 receptor expression: intelligent design. Nature

393 Reviews Immunology 7:144-154

394 Miyasaka M, Tanaka T (2004) Lymphocyte trafficking across high endothelial venules: dogmas and 395 enigmas. Nat Rev Immunol 4: 360-370

396 Moro K, Yamada T, Tanabe M, Takeuchi T, Ikawa T, Kawamoto H, Furusawa J, Ohtani M, Fujii H, 397 Koyasu S (2010) Innate production of TH2 cytokines by adipose tissue-associated c-Kit ${ }^{+} \mathrm{Sca}-1^{+}$ 398 lymphoid cells. Nature 463: 540-544

Murray AB, Luz A (1990) Acidophilic macrophage pneumonia in laboratory mice. Vet Pathol 27:

Nair PN, Zimmerli I, Schroeder HE (1987) Minor salivary gland duct-associated lymphoid tissue (DALT) in monkeys changes with age. J Dent Res 66: 407-411

Nishikawa S, Honda K, Vieira P, Yoshida H (2003) Organogenesis of peripheral lymphoid organs.

Shan Y, Liu J, Jiang YJ, Shang H, Jiang D, Cao YM (2012) Age-related susceptibility and resistance 
Shimizu S, Sugiyama N, Masutani K, Sadanaga A, Miyazaki Y, Inoue Y, Akahoshi M, Katafuchi R,

411 glomerulonephritis development with Th2-type immune deviations in MRL/lpr mice deficient for

IL-27 Receptor (WSX-1). J Immunol 175: 7185-7192

414 NKp46(+) natural killer cells in the intestinal mucosa of patients with Crohn's disease.

415 Gastroenterology 139: 882-892

416 Takemura S, Braun A, Crowson C, Kurtin PJ, Cofield RH, O’Fallon WM, Goronzy JJ, Weyand CM

417 (2001) Lymphoid neogenesis in rheumatoid synovitis. J Immunol 167: 1072-1080

418 Taupin P (2007) BrdU immunohistochemistry for studying adult neurogenesis: paradigms, pitfalls, limitations, and validation. Brain Res Rev 53: 198-214

Tilg H, Moschen AR (2006) Adipocytokines: mediators linking adipose tissue, inflammation and immunity. Nat Rev Immunol 6: 772-783 
Fig. 1 Gross anatomical localization of mediastinal fat tissue (MFT) in mice: White-colored MFT (arrows) extending from the diaphragm (D) to the heart (H). DBA/2Cr mouse, 12 months. C: chest including sternum and ribs. T: thymus

Fig. 2 Mediastinal fat-associated lymphoid clusters in mice. Panels a-f show the stereomicroscopic photographs of whole-mount hematoxylin-stained mediastinal fat tissues (MFTs) from C57BL/6N (panels A and D), DBA/2Cr (panels B and E), and MRL/MpJ (panels $\mathrm{C}$ and $\mathrm{F}$ ) mice at 12 months. The squares in panels a-c indicate the same areas as those in panels $d-f$. In each panel, the dark-stained regions represent the lymphoid clusters (LCs) of the MFT (panels d-f, arrows). Larger numbers and areas of LCs are visible in C57BL/6N mice (panels a and d) compared with DBA/2Cr (panels $\mathrm{b}$ and e) and MRL/MpJ (panels c and f) mice. (g) Percentage of LC area in the total MFT area in the hematoxylin whole-mount specimens. D and M: Significant differences between DBA/2Cr (D) and MRL/MpJ (M) mice, analyzed by Tukey's post-hoc test subsequent to ANOVA ( $p$ $<0.05$ ); $\mathrm{n} \geq 3$ mice of each strain (at 3 months $\mathrm{n}=5,3,3$ and at 12 months $\mathrm{n}=5,5,5$ in C57BL/6N, $\mathrm{DBA} / 2 \mathrm{Cr}$, and $\mathrm{MRL} / \mathrm{MpJ}$ mice, respectively). Values are given as the mean $\pm \mathrm{SE}$

Fig. 3 Histological features of mediastinal fat-associated lymphoid clusters in mice. Panels a-f show 
443 light microscope photographs of hematoxylin and eosin (HE)-stained mediastinal fat tissue (MFT)

444 sections of C57BL/6N (panels a and d), DBA/2Cr (panels b and e), and MRL/MpJ (panels c and f)

445 mice at 12 months. The squares in panels a-c indicate the same areas as those in panels $\mathrm{d}-\mathrm{f}$. In each

446 panel, the accumulation of mononuclear cells is visible in the MFT (panels d-f). More lymphoid

447 clusters (LCs) with larger areas are visible in C57BL/6N mice (panels a and d) compared with

$448 \mathrm{DBA} / 2 \mathrm{Cr}$ and MRL/MpJ mice. (g) Percentage of LC area in the total MFT area in the HE-stained

449 sections. D and M: Significant differences between DBA/2Cr (D) and MRL/MpJ (M), analyzed by

450 Tukey's post-hoc test subsequent to ANOVA ( $p<0.05) ; \mathrm{n} \geq 3$ mice of each strain (at 3 months $\mathrm{n}=5$,

4514,3 and at 12 months $\mathrm{n}=4,4,4$ in $\mathrm{C} 57 \mathrm{BL} / 6 \mathrm{~N}, \mathrm{DBA} / 2 \mathrm{Cr}$, and $\mathrm{MRL} / \mathrm{MpJ}$ mice, respectively). Values

452 are given as the mean $\pm \mathrm{SE}$

Fig. 4 Blood vessels in mediastinal fat-associated lymphoid clusters (MFALCs) in mice. Panels a and $b$ show light microscope photographs of hematoxylin and eosin (HE)-stained mediastinal fat tissue (MFT) sections of C57BL/6N mice at 12 months. Large blood vessels containing lymphocytes (arrowheads) are visible (panel a). In the MFALCs, the endothelial cells of the vessels show epithelioid features with abundant cytoplasms and large nuclei (panels a and b). The squares in panel a indicate the same areas as those in panel b. Panels c-e show light microscope photographs of 
(panel c), DBA/2Cr (panel d), and MRL/MpJ mice (e) at 12 months. PNAd-positive endothelial cells

$\mathrm{f}-\mathrm{h}$ show the serial section of panels c-e stained by normal rat IgM instead of anti-PNAd antibody,

and no normal IgM-positive reaction is observed in the venules.

Fig. 5 Lymphatic vessels in mediastinal fat-associated lymphoid clusters in mice. Panels a-f show

light microscope photographs of immunohistochemical stains for the lymphatic vessels marker

LYVE-1 in C57BL/6N (panels a-c), DBA/2Cr (panel d), and MRL/MpJ mice (e and f) at 12 months.

The solid and dotted squares in panel a indicate the same areas as those in panel b and c respectively.

LYVE-1-positive lymph vessels are clearly observed in the mediastinal fat tissues (MFTs) (panel b

e indicate the same area as that in panel f. LYVE-1-positive lymphatic vessels were well developed mice (e and f).

Fig. 6 Immune cell populations of mediastinal fat-associated lymphoid clusters (MFALCs) in mice.

Panels a-h show light microscope photographs of immunohistochemical stains for B220 (B-cells, 

the MFALC of C57BL/6N mice. (i) Index of immune cell populations in the MFALCs of the mice. a, strain, m: Significant difference with $\mathrm{MRL} / \mathrm{MpJ}$ in same antigen, analyzed by Tukey's post-hoc test

Fig. 7 CD133-positive cells of mediastinal fat-associated lymphoid clusters (MFALCs) in mice.

Panels a-d show light microscope photographs of immunohistochemical stains for CD133 (marker

for hematopoietic progenitor cells)-positive cells in C57BL/6N mice at 12 months. The dotted and solid squares in panel $\mathrm{b}$ indicate the same areas as those in panel $\mathrm{c}$ and $\mathrm{d}$ respectively. Few positive cells are visible in the MFALC (panel a, arrows) and in the lumen of the MFALC vessels (v) (panel b and c). In panel d, the smaller-sized lymphoid clusters showed numerous CD133-positive cells. Panels e and $\mathrm{f}$ are stained by normal rabbit IgG instead of anti-CD133 antibody for negative control, and the square in panel e indicate the same area as those in panel f. No normal IgG-positive cells were observed in the MFALC (Panels 7e and f).

Fig. 8 c-Kit- and CD127-positive cells of mediastinal fat-associated lymphoid clusters (MFALCs) in 
497 mice. Panels are representative images of immunofluoresce for c-Kit (panel a, green) and CD127

498 (panel b, red) with nuclear staining by Hoechst (panel c, blue) in C57BL/6 at ** months. Merged

499 image is shown in panel d, demonstrating the presence of few c-Kit $+/ \mathrm{CD} 127+$ cells (arrows) and 500 many c-Kit-/ CD127+ cells within the MFALC.

Fig. 9 Proliferating cells in the mediastinal fat-associated lymphoid clusters (MFALCs) in mice.

Proliferating cells were detected by immunohistochemistry for $\mathrm{BrdU}$ in $\mathrm{DBA} / 2 \mathrm{Cr}$ at 12 months

(panel a) and C57BL/6N mice at 3 and 12 months (panels b and c respectively). Panels $d$, e and $f$ are

higher magnification to the square area in panels $\mathrm{a}, \mathrm{b}$ and $\mathrm{c}$ respectively. Note fewer BrdU-positive

cells are visible within the MFALCs in DBA/2Cr mice (panel a and d) compared to C57BL/6N mice observed at 12 months (panels b and e) compared to 3 months (panels $\mathrm{c}$ and f). 
Figure 1.

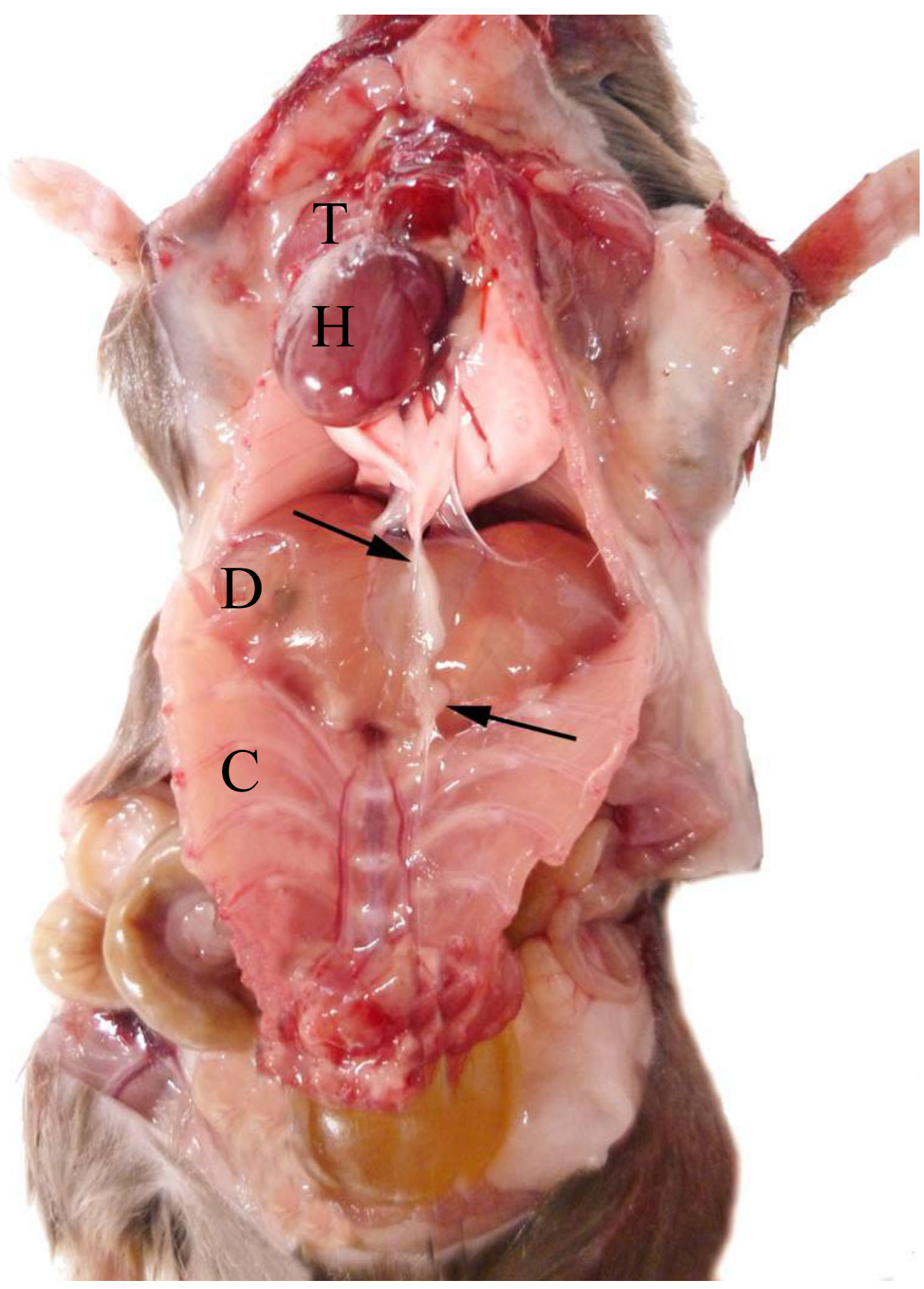


Figure 2.
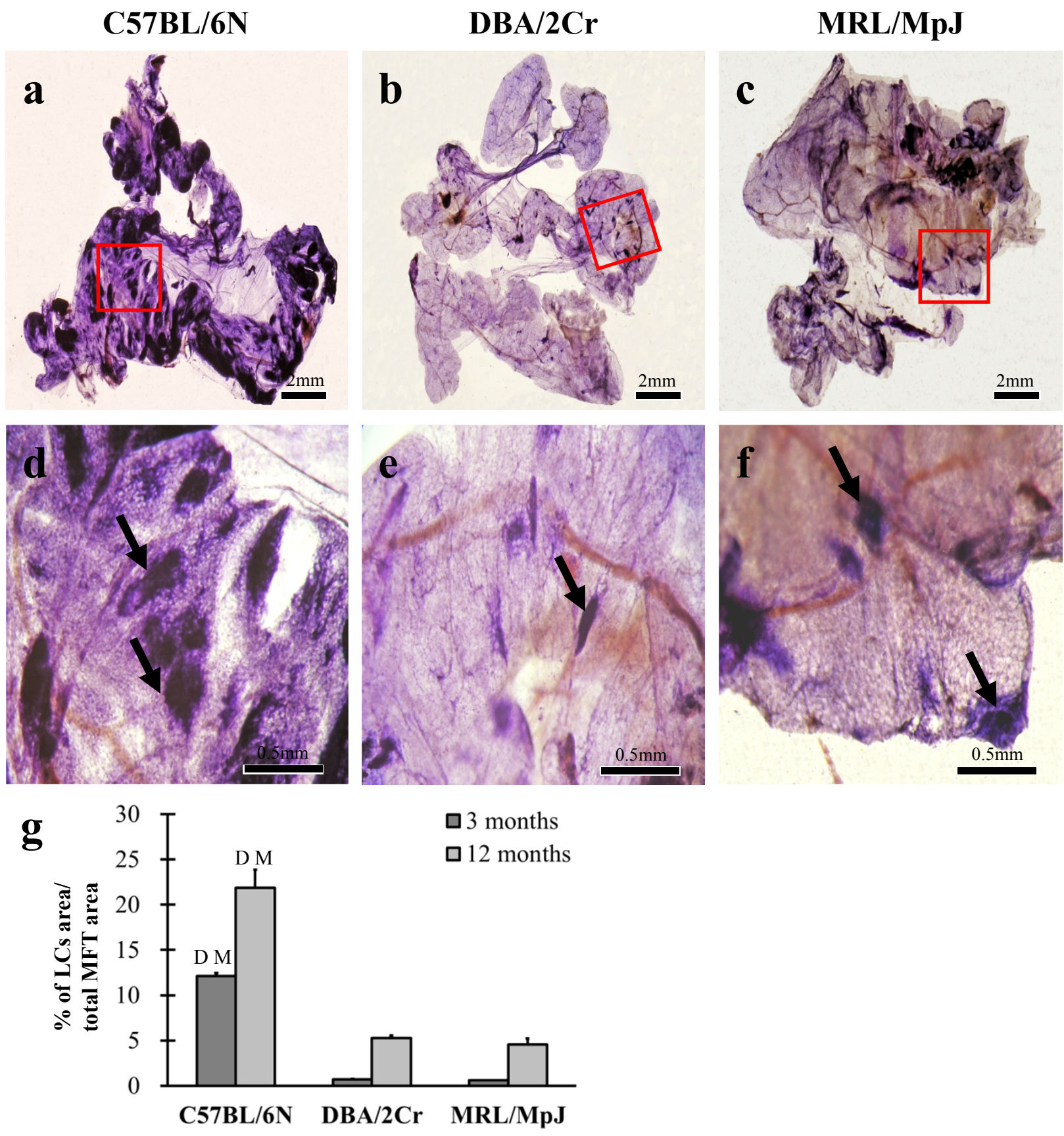
Figure 3.

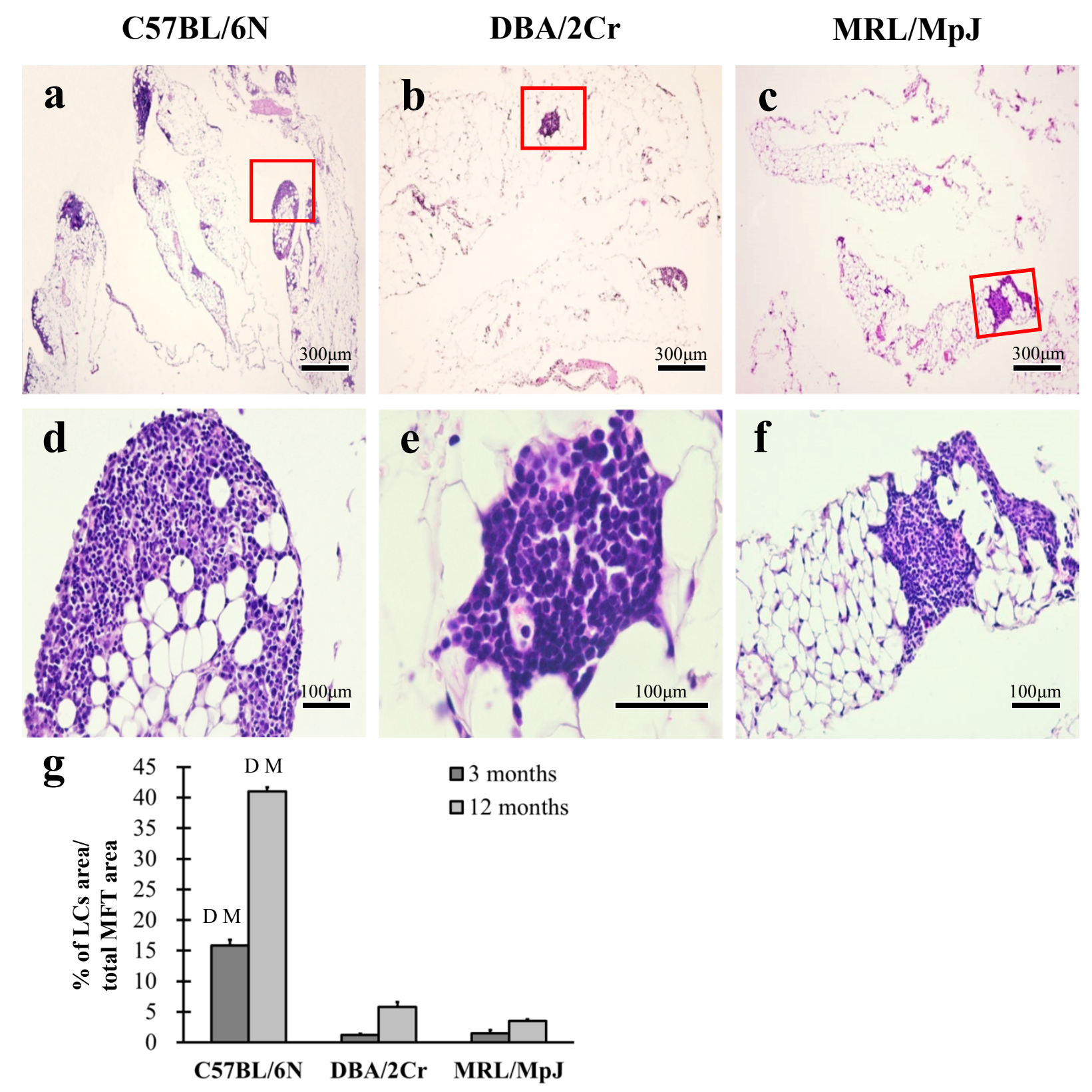




\section{Figure 4.}
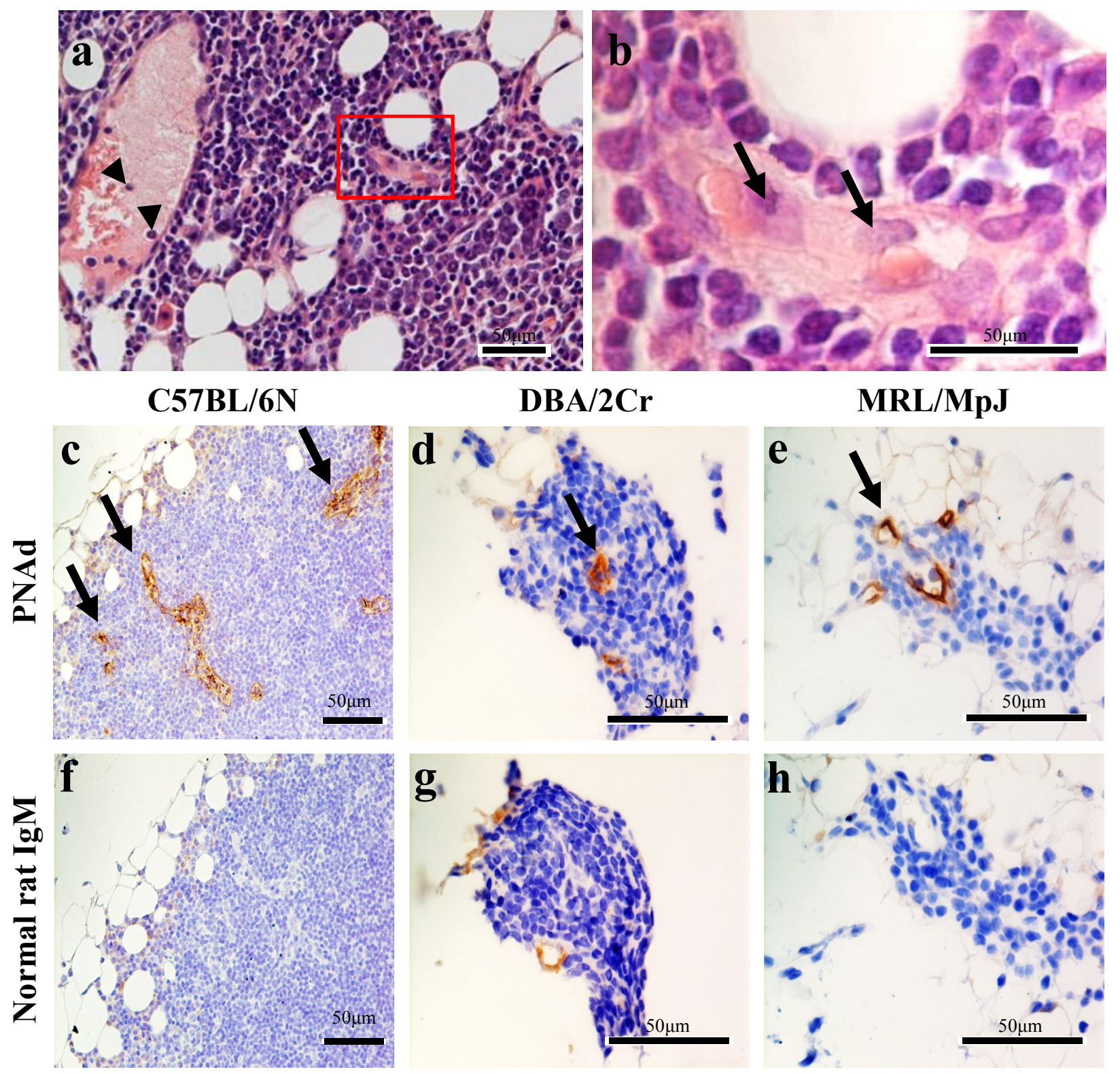
Figure 5.

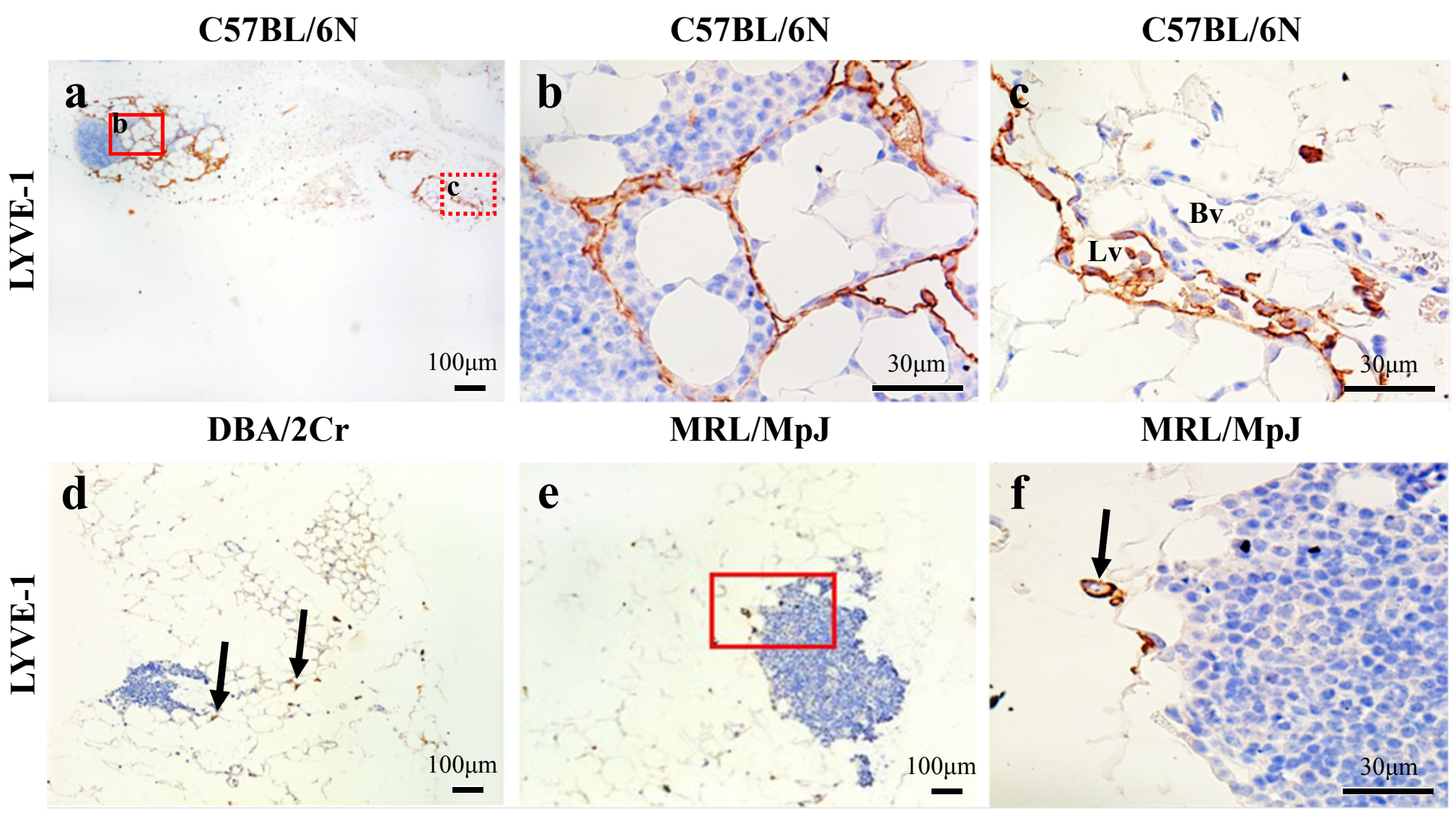




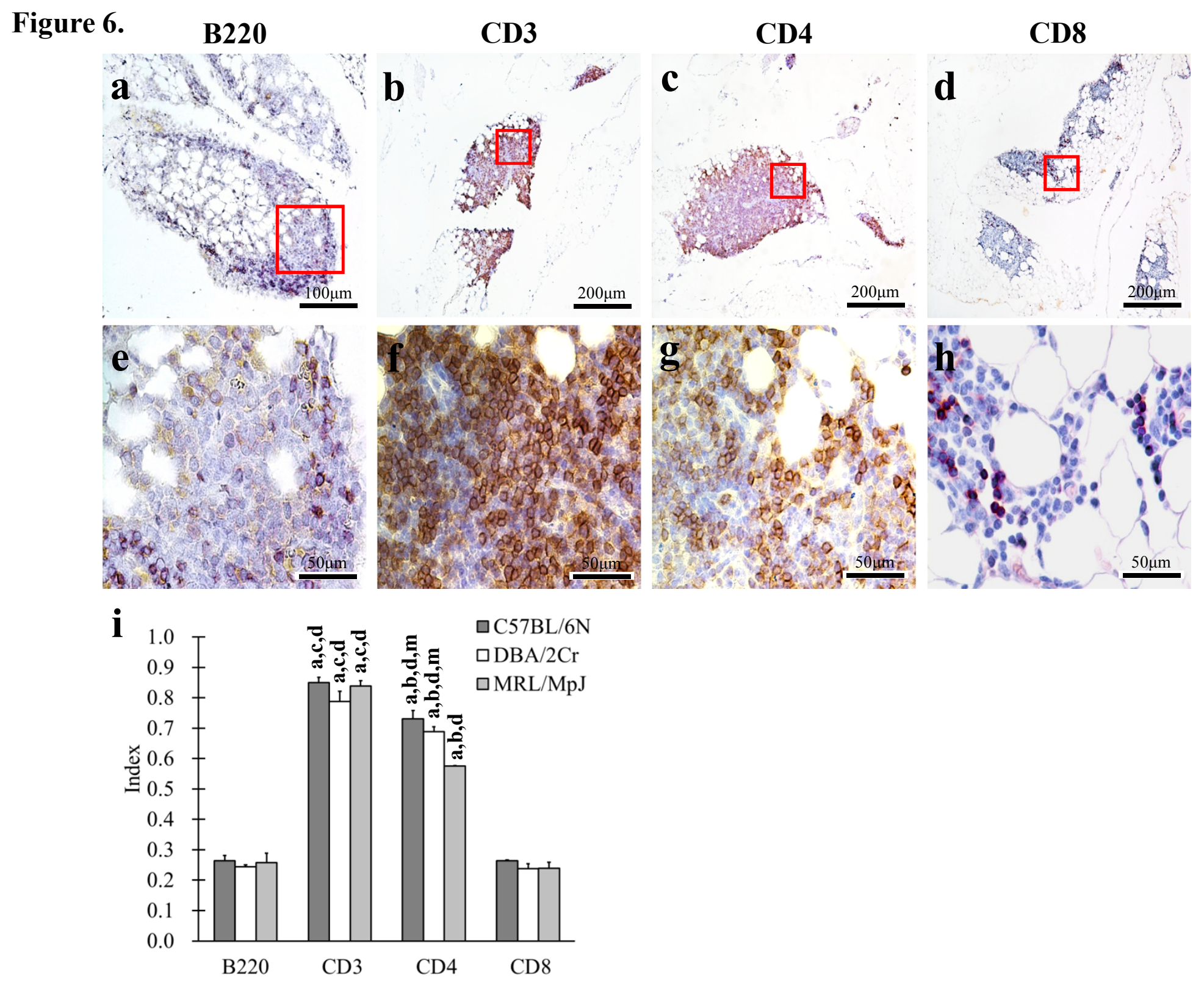


Figure 7.

Figure 8.

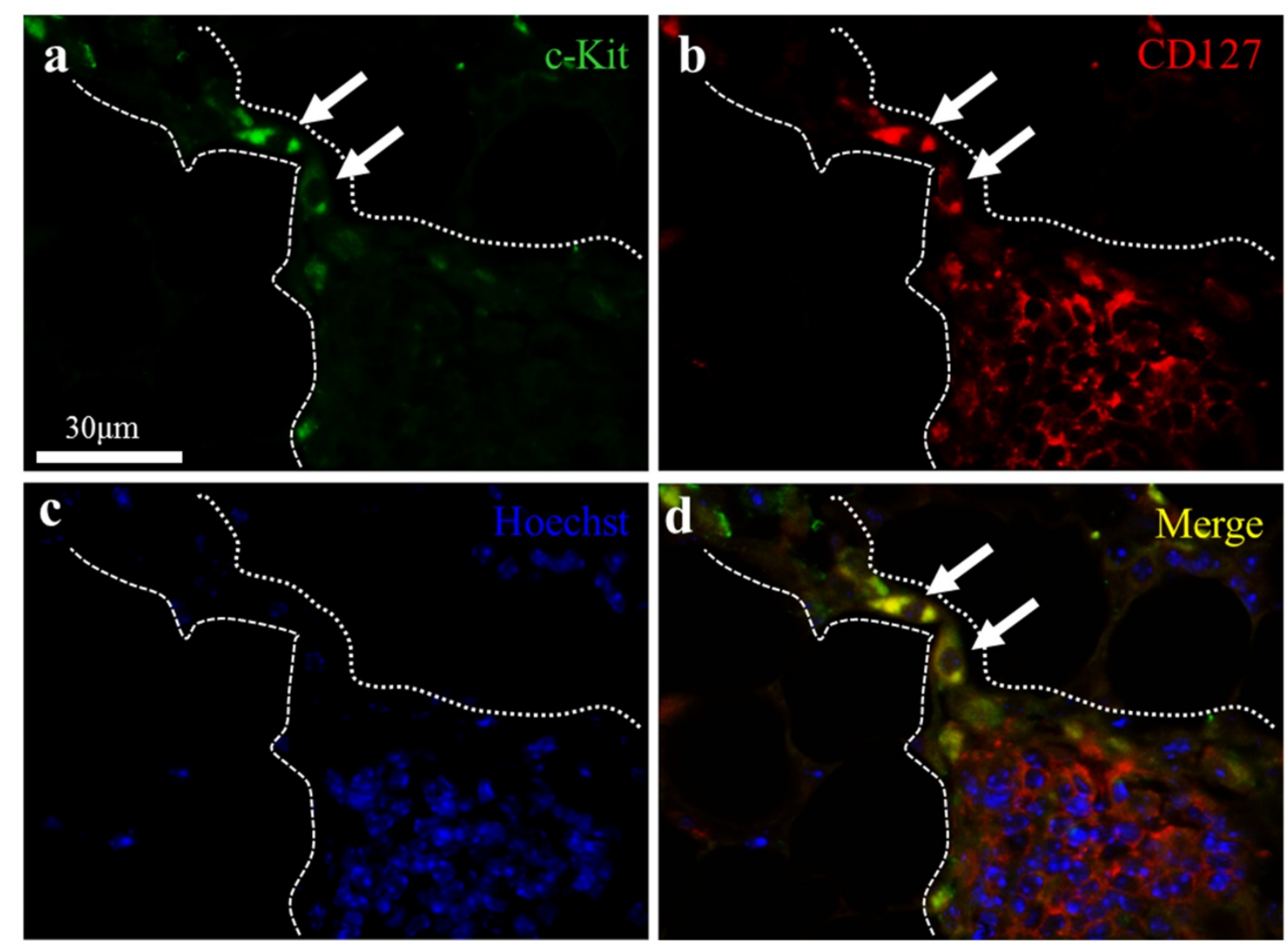




\section{Figure 9.}

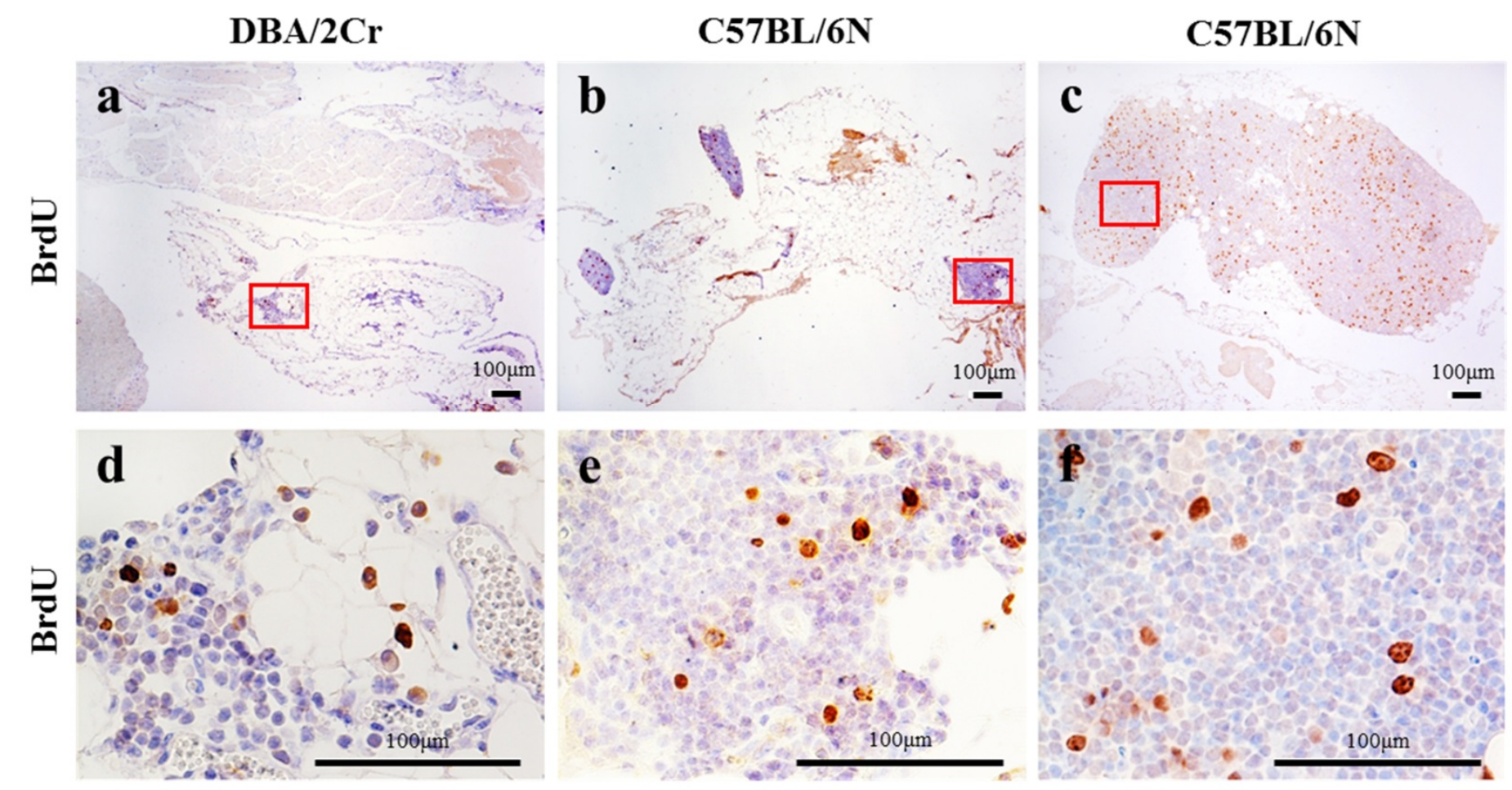

